\title{
Interhemispheric asymmetry of electrical activity of the brain in sleep and "cerebral dominance"
}

\author{
MICHAEL S. MYSLOBODSKY, VARDA BEN-MAYOR, BATIA YEDID-LEVY, and MATTI MINZ \\ Neurobiology Unit, Tel-Aviv University, Ramat-Aviv, Israel
}

\begin{abstract}
Monopolar recordings of the EEG during sleep from each occipital area in normal volunteers indicated a consistent prevalence of spindles over the right hemisphere and more pronounced slow wave activity over the left. Asymmetry in the slow wave activity was confirmed by an analysis of the secondary components of the visually evoked potentials. The data indicated that the hemisphere generally considered to be "subdominant" during the awake state is subject to less intense non-REM sleep, suggesting a reversal in the "dominance" of hemispheres in sleep.
\end{abstract}

It is recognized that in humans each cerebral hemisphere has distinctive functions beyond those related to the simple control of the sensory and motor activities of the body. Thus, language-related functions have been shown to depend upon left hemispheric activity while the right hemisphere is believed to be involved in visuo-spatial problem solving (Dimond \& Beaumont, 1974).

This functional specialization of hemispheres is reflected in the asymmetry of the EEG and in some components of evoked potentials (Galin \& Ellis, 1975; Myslobodsky, 1973; Wood, Gold, \& Day, 1971). Furthermore, both spontaneous and evoked brain activity may be unilaterally affected in a predictable task-specific way by selectively facilitating the activity of one or the other hemisphere.

To date, the functional asymmetry of the human brain has been inferred exclusively from studies conducted in the waking state. There is limited information indicating that the differences in hemispheric activity observed in the waking brain might also be a characteristic of sleep (Giannitrapani, Gorkin, \& Enenstein, 1966; Goldstein, Stoltzfus, \& Gardocki, 1972). Some writers believe that asynchronous sleep patterns are normal up to 5 months of age, but thereafter interhemispheric EEG asynchrony is an abnormal feature (Garvin \& Gibbs, 1975). However, if certain signs of waking EEG asymmetry are associated with specific hemispheric functions, then the peculiar type of mentation, perception, learning, and responding occurring in sleep would be expected to markedly affect interhemispheric functional relations. The purpose of the present study was to explore this question.

This research was supported in part by the Gorovitz Grant to M.S.M. The authors wish to thank Dr. N. Friedland for his help with the statistical treatment of the data. The paper was sponsored by Irving Maltzman, who takes full editorial responsibility for its contents. Reprint requests should be sent to the first author. Neurobiology Unit, Department of Psychology. Tel-Aviv University, Ramat-Aviv, Tel-Aviv, Israel.

\section{METHOD}

\section{Subjects}

Eight healthy adult student volunteers participated in this investigation. Only right-handed students were selected. In four students. undisturbed night sleep EEG records were analyzed. and in four students. visual evoked potentials were studied (VEP). All students came to the laboratory at about their normal bedtime.

\section{Procedure}

EEG was recorded from bilaterally symmetrical points of the occiput. $2.5 \mathrm{~cm}$ laterally and $2.5 \mathrm{~cm}$ above the inion. Joined mastoids sered as a reference. Special care was taken to maintain identical resistance of the electrodes and characteristics of both amplifying and recording systems. EEG and EOG were recorded in all cases for REM sleep identification. Sleep was staged using the criterial of Rechtshaffen and Kales (1968).

VEP were recorded monopolarly from the same EEG electrodes. Photic stimuli generated by a xenon discharge tube were delivered aperiodically for 20-40 min prior to sleep and then in different sleep stages at a rate of approximately about .3 /second. The distance between the tube and the nose bridge was about $50 \mathrm{~cm}$. The direction of the flash changed with variations of the head position. The EEG was analyzed by a computer of averaged transients and recorded on an X-Y plotter. No more than 20 responses were averaged in sleep. to avoid possible contamination of the VEP with occasional movement artifacts. In this study, only data on non-REM sleep were collected. In EEG analisis. only qualitative (pattern) hemispheric differences were taken into consideration and estimated for further processing. For the estimation of the amount of slow wave activity in the EEG. the area of the VEP secondary potential (slow negative wave) was planimetrically measured. A detailed justification of this approach to the analysis of human sleep was published elsewhere (Myslobodsky. 1973).

\section{RESULTS}

Visual inspection of sleep records revealed that electrical activity was asymmetrical in all subjects. The most consistent and clear asymmetry was found in spindle activity. The latter was always more prominent over the right hemisphere. In the left hemisphere, such activity was frequently absent or modulated by slower background activity. The right hemisphere also showed a greater amount of spindles for the entire night sleep in a group of subjects $[\mathrm{t}(3)=3.14, \mathrm{p}<.05]$. 


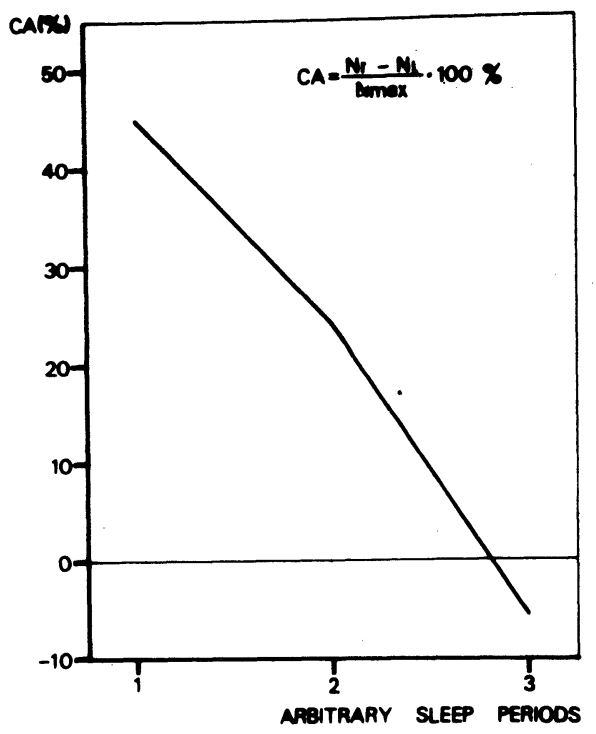

Figure 1. Interhemispheric differences in the amount of spindles during night sleep. Averaged data for three subjects. Ordinate indicates asymmetry coefficient (CA); abscissa indicates arbitrary sleep periods, total time spent in sleep divided into three equal time periods.

Right hemisphere spindle predominance was independent of sleep stages. For this reason, sleep records were subdivided into three equal time periods ("arbitrary sleep periods") to facilitate the processing and presentation of the data. Analysis revealed that the predominance of spindles over the right hemisphere was inversely correlated with the total amount of time spent in sleep. In Figure 1, this result is presented as a coefficient of asymmetry (CA) of the spindle number

$$
\left(C A=\frac{n_{r}-n}{n_{\max }} 100 \%\right),
$$

where $\mathrm{n}$ is a number of spindles over the right or left side. This asymmetry was significant for the arbitrary first $[t(3)=5.34, p<.01]$ and nonsignificant for the second period $[t(3)=1.93]$. The amount of spindles in both hemispheres gradually increased throughout the night, especailly from the second to the third period. In the left hemisphere, this trend was more pronounced $[F(1,3)=25.94, p<.05]$ and the $C A$ progressively diminished to the end of sleep, becoming nonsignificant in the third period (Figure 1).

One sees such dynamics when subjects sleep without awakenings. During the sleep which immediately follows an awakening, one can usually see an abrupt return of the CA to its initial values. It seems as if spindle asymmetry is associated with certain processes responsible for the onset of sleep, and the loss of asymmetry reflects the amount of sleep time spent without awakenings.

As mentioned above, in cases of right occiput spindling in the absence of corresponding left spindling, the left occiput showed low-voltage slow activity in the theta range, or low-amplitude disorganized spindles superimposed on the slow activity. This was a consistent finding in all subjects $[t(3)=2.79, \quad p<.05]$, suggesting that the left hemisphere displays a more advanced stage of non-REM sleep.

This conclusion was checked by comparing hemispheric reactivity to photic stimuli, by analyzing VEPs. Presleep human VEPs are composed of a series of positive-negative waves terminating with oscillations in the alpha frequency range (alpha afterdischarge [Myslobodsky, 1973]). The most unambiguous qualitative VEP changes during sleep occur in this secondary component of the response and in the wave preceding it (N4 wave), which may be considered to be the initial component of the alpha afterdischarge.

In Stage I of sleep, the alpha afterdischarge is suppressed and the $\mathrm{N} 4$ wave facilitated. In the subsequent Stages II-III, the N4 wave progressively increases in amplitude and duration until in Stage IV it finally displaces and occupies the section previously occupied by the alpha afterdischarge.

On the other hand, the N4 wave was shown to be suppressed with the initiation of REM sleep (Shagass \& Trusty, 1966; Myslobodsky, 1973). Hence the duration, amplitude, or planimetrically measured area of this secondary component can be used as an index of the degree of EEG synchronization or of the amount of slow activity in different stages of sleep.
Figure 2. The reversal of the coefficient of asymmetry (CA) in VEP secondary components during sleep. In $A$, the dynamics of $\mathrm{CA}$ are indicated by columns plotted againat different sleep-stages in $B$, when the VEP records were taken. Solid columns and lines in $A$ and $B$ indicate the first and the seond night data in the same subject. Note prealeep level eatimated for the area of N4 alpha-afterdischarge components of VEP.

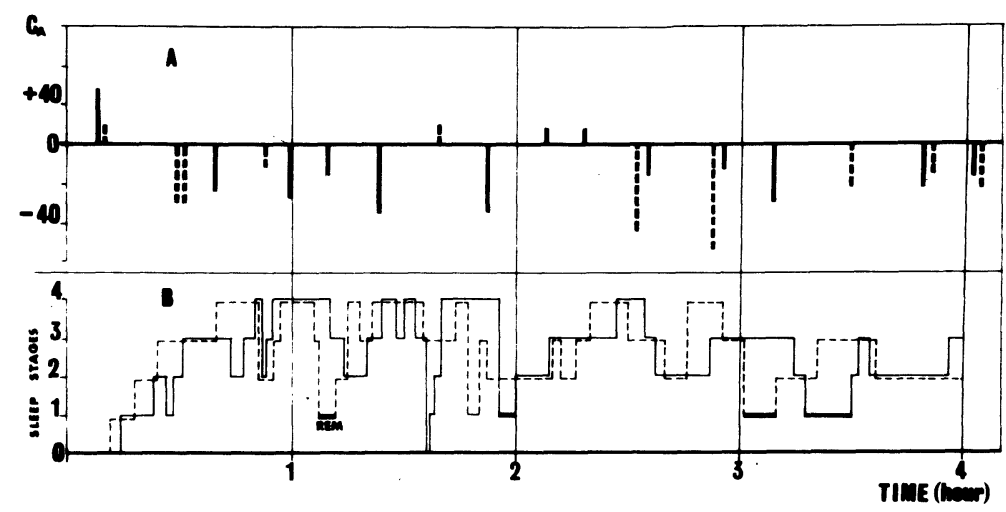


Presleep VEP analysis showed that slight and variable asymmetry was evident only in the alpha afterdischarge portion of the VEP, which was shown to be slightly higher (about $20 \%-25 \%$ ) over the right occiput. In this study, a right .hemispheric predominance of the afterdischarge was statistically significant only in one subject $[t(15)=2.13$, $\mathrm{p}<.02]$.

With falling asleep, a radical VEP metamporphosis occurred in both hemispheres, as described above. However, the asymmetry of the secondary VEP component became evident as soon as the subject displayed the first sign of sleep. This asymmetry was in the same direction in all four subjects studied and was statistically significant for this group [ $\mathrm{t}(3)=$ $2.96, \mathrm{p}<.05]$.

The asymmetry coefficient of the area of the secondary slow negative wave of the VEP was calculated in the same way as it was for the estimation of spindle asymmetry. In Figure 2, the values of this coefficient are plotted against different stages of sleep. The CA of the alpha afterdischarge area was estimated in order to serve as a reference for further comparison with corresponding sleep data. It is quite clear that in the presleep state, the CA had a positive sign (predominance of the afterdischarge over the right occiput), while with the advent of sleep the sign of the CA was reversed due to the predominance of the amplitude and duration of the slow secondary negative wave over the left side. This reversal obtained during all stages of sleep and could be seen as long as 10-20 min after awakening even though the form of the VEP at this time was close to that of the presleep stage.

\section{DISCUSSION}

It has frequently been reported that alpha rhythm and alpha afterdischarge of VEP are more pronounced over the right hemisphere (see Galin \& Ellis, 1975). Writers display a rare unanimity about the meaning of alpha. From the first, it was recognized to be an indicator of rest. relaxation. lowered attention, and arousal level-in short. an inhibitory phenomenon. Hence the relative predominance of alpha on the right may be considered a symptom indicating that the right hemisphere in right-handed persons operates in a somewhat inhibitory state. or that the left hemisphere is the "wakefulness-dominant" hemisphere. According (1) the same logic, the results reported here suggest the consideration of the right hemisphere as "sleep dominant."

There are some reasons to speculate that this day-night interchange of dominance reflects certain basic adaptive mechanisms controlling the emotional charge of the right hemisphere. Neurologists have long encountered cases of depressive-catastrophic reactions in patients with defects of the left hemisphere and reactions of indifference, euphoria, and anosognosia with lesions of the right brain (Gainotti, 1972). In the went of pharnacological inactivation of the left hemisphere (homolateral intracarotid amytal injections) depressive reactions were seen. whereas the injection of the amytal into the right carotid induced a state of euphoria (Terzian. 1964). It is attractive to speculate that this predominance of right hemispheric activity is the basis for usually negative dream content (Mack, 1974) and that in some pathological conditions or in predisposed individuals this sleep-dominant night hemispheric activity may be the source of arousal with highly abnormal mental activities such as night terrors. Indeed, the latter were shown to arise out of slow wave sleep usually during the first non-REM periods of night (Broughton. Poire, \& Tassinari. 1965; Fisher, Byrne, \& Edwards, 1968). They may be interpreted as the equivalent of depressive-catastrophic reactions due to the release of the right hemisphere after left-side amytal injection or left hemispheric lesion. Vigorous GSR ("GSR storms") recorded during slow wave sleep, especially Stages III and IV (Broughton, Poire, \& Tassinari, 1965) are believed to reflect this relative predominance of the right hemisphere activity. In a previous study (Myslobodsky \& Rattok, 1975), it was shown that right-hemisphere-mediated orienting responses are accompanied by a greater amount of electrodermal activity than left-hemisphere mediated orienting responses.

\section{REFERENCES}

Broughton, R. J., Poire, R., \& Tassinari, C. A. The electrodermogram (Tarchanoff effect) during sleep. Electroencepalography and Clinical Neurophysiology, 1965, 18, 691-708.

Dimond, S. J., \& Beaumont, J. G. (Eds.), Hemispheric function in the human brain. New York: Wiley, 1974.

Fisher, C., Byrne, J., \& Edwards, A. (1968) cited by Mack, J. Nightmares and human conflict. Boston: Houghton Mifflin, 1974. P. 18.

Gainotti, G. Emotional behavior and hemispheric side of the lesion. Cortex, 1972, 8, 41-55.

Galin, D., \& Ellis, R. R. Asymmetry in evoked potentials as an index of lateralized cognitive process: Relation to EEG alpha asymmetry. Neuropsychologia, 1975. 13. $45-50$.

GaRVIN, J. S., \& GibBs, E. L. Electroencephalogram in hydrocephalus. Clinical Electroencephalography, 1975, 6. 141-144.

Giannitrapani, G., Gorkin, A., \& Enenstein, J. Laterality preference of children and adults as related to interhemispheric EEG phase activity. Journal of Neurological Science, $1966,3,139-150$.

Goldstein, L., Stoltzfus, N., \& Gardocki, J. F. Changes in interhemispheric amplitude relationships in the EEG during sleep. Physiology and Behavior, 1972, 8, 811-815.

MACK, J. Nightmares and human conflict. Boston: Houghton Mifflin, 1974.

MysLOBODSKY, M. Hypersynchronous activity of the cerebral cortex. Moscow: "Nauka," 1973.

Myslobodsky, M., \& Rattock. J. Asymmetry of electrodermal activity in man. Bulletin of the Psychonomic Society, 1975, 6, 501-502.

Rechtschaffen, A., \& Kales, A. (Eds.), A manual of standardized terminology, techniques and scoring system for sleep stages of human subjects. National Institute of Health, 204, Washington, D.C: U.S. Government Printing Office, 1968.

Shagass, C., \& Trusty, D. Somatosensory and visual cerebral evoked responses changes during sleep. Recent advances in biological psychiatry, 1966, 8, 321-334.

TERzIAN, H. Behavioral and EEG effects of intracarotid sodium amytal injection. Acta Neurochirurgica, 1964, 12, 230-239.

Wood, C., Gold, W., \& DAY, R. Auditory evoked potentials during speech perception. Science, 1971, 173, 1248-1251.

(Received for publication February 3, 1976.) 